\title{
Greater functional aerobic capacity predicts more effective pain modulation in older adults
}

\author{
Tom Ohlman ${ }^{1}$, Keith Naugle ${ }^{1}$, NiCole Keith ${ }^{1}$, Zachary Riley ${ }^{1}$, Kelly M. Naugle ${ }^{1}$ \\ 1:Department of Kinesiology, School of Physical Education and Tourism Management, \\ Indiana University-Purdue University Indianapolis
}

Endogenous pain inhibitory and facilitory function deteriorates with age, potentially placing older adults at greater risk for chronic pain. Prior research shows that self-reported physical activity predicts endogenous pain inhibitory capacity and facilitation of pain on quantitative sensory tests (QST) in healthy adults. Purpose: To investigate whether functional aerobic capacity and lower extremity strength in older adults cross-sectionally predicts pain sensitivity, pain inhibition following isometric exercise, and facilitation of pain during heat pain temporal summation (TS) tests. Methods: 42 subjects (10 male, 32 female, age $=67.5 \pm 5.1$ ) completed the 6-minute walk test (6MWT), 30-second chair stand test, and several QSTs. QSTs included: 1) Pain ratings (0-100 scale) during the submersion of the hand in a cold water bath (CWB), 2) heat pain threshold tests, 3) the amount of pain reduction following submaximal isometric exercise, and 4) degree of pain facilitation during temporal summation tests conducted at 44,46 , and $48^{\circ} \mathrm{C}$. Responses on the QSTs were analyzed using hierarchical linear regression with meters on 6MWT and number of chair stands as final predictors. Results: After controlling for demographic and psychological factors, aerobic capacity on 6MWT significantly predicted CWB pain ratings $(\mathrm{R} 2$ change $=22.5 \%$, Beta $=-0.491)$, pain facilitation during $\mathrm{TS}$ trials at $44^{\circ} \mathrm{C}(\mathrm{R} 2$ change $=$ $16.7 \%$, Beta $=-0.446$ ), and the amount of pain reduction following isometric exercise (R2 change $=20.7 \%$, Beta $=0.393)$. All other analyses were not significant $(\mathrm{P}>0.05)$.

Conclusions: Older adults exhibiting greater functional aerobic capacity displayed reduced cold pain sensitivity, reduced pain facilitory function, and increased pain inhibition following exercise. These findings suggest that increased aerobic fitness in older adults may be associated with more effective endogenous modulation of pain. This study was funded by the IUPUI School of PETM Faculty Research Opportunity Grant. 